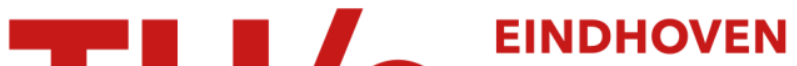 UNIVERSITY OF TECHNOLOGY
}

\section{Electric-field gradients in thin face-centered-tetragonal Co films observed by nuclear magnetic resonance}

\section{Citation for published version (APA):}

Wieldraaijer, H., Jonge, de, W. J. M., \& Kohlhepp, J. T. (2005). Electric-field gradients in thin face-centeredtetragonal Co films observed by nuclear magnetic resonance. Physical Review B, 72(15), 155409-1/10. [155409]. https://doi.org/10.1103/PhysRevB.72.155409

DOI:

10.1103/PhysRevB.72.155409

Document status and date:

Published: 01/01/2005

\section{Document Version:}

Publisher's PDF, also known as Version of Record (includes final page, issue and volume numbers)

\section{Please check the document version of this publication:}

- A submitted manuscript is the version of the article upon submission and before peer-review. There can be important differences between the submitted version and the official published version of record. People interested in the research are advised to contact the author for the final version of the publication, or visit the $\mathrm{DOI}$ to the publisher's website.

- The final author version and the galley proof are versions of the publication after peer review.

- The final published version features the final layout of the paper including the volume, issue and page numbers.

Link to publication

\section{General rights}

Copyright and moral rights for the publications made accessible in the public portal are retained by the authors and/or other copyright owners and it is a condition of accessing publications that users recognise and abide by the legal requirements associated with these rights.

- Users may download and print one copy of any publication from the public portal for the purpose of private study or research.

- You may not further distribute the material or use it for any profit-making activity or commercial gain

- You may freely distribute the URL identifying the publication in the public portal.

If the publication is distributed under the terms of Article 25fa of the Dutch Copyright Act, indicated by the "Taverne" license above, please follow below link for the End User Agreement:

www.tue.nl/taverne

Take down policy

If you believe that this document breaches copyright please contact us at:

openaccess@tue.nl

providing details and we will investigate your claim. 


\title{
Electric-field gradients in thin face-centered-tetragonal Co films observed by nuclear magnetic resonance
}

\author{
H. Wieldraaijer, W. J. M. de Jonge, and J. T. Kohlhepp* \\ Department of Applied Physics, Center for Nanomaterials (cNM) and COBRA Research Institute, Eindhoven University of Technology, \\ P.O. Box 513, 5600 MB Eindhoven, The Netherlands
}

(Received 2 May 2005; revised manuscript received 12 July 2005; published 12 October 2005)

\begin{abstract}
Thin tetragonally strained fcc-Co films grown epitaxially on $\mathrm{Cu}(001)$ single crystals have been investigated by ${ }^{59} \mathrm{Co}$ nuclear magnetic resonance (NMR). The ultrahigh structural quality and the homogeneity of the strain result in the direct NMR observation of electric-field gradients, which is unique in thin films. The structural quality and the homogeneity and size of the strain are analyzed by means of the very small NMR linewidths, the hyperfine field anisotropies, and the uniform electric-field gradients. A quantitative agreement with the observed strain is found. The strain, and thus the tetragonal distortion, in the films is found to be surprisingly stable, showing less than $10 \%$ strain relief for 80 ML Co films.
\end{abstract}

DOI: 10.1103/PhysRevB.72.155409

PACS number(s): 68.55.Jk, 76.60.-k, 62.20.Dc

\section{INTRODUCTION}

The physical structure of thin films generally shows significant deviations from that of the bulk material. An example of this is the strain arising from the different lattice parameters of the film material and the substrate. In magnetic thin films, which are of strong interest due to the use of these films in various spintronic devices or model systems, ${ }^{1,2}$ the magnetic and electronic properties may be strongly influenced by this strain and other structural aspects. This influence makes it important to accurately investigate the specific physical structure of thin films.

A model system for this kind of investigation is Co grown on $\mathrm{Cu}(001)$. Under proper conditions, the $\mathrm{Co}$ film has been shown to grow in an almost layer-by-layer fashion and to form a face-centered-tetragonal (fct) structure of high quality over a reasonable thickness range. ${ }^{3-7}$ This fct-Co phase is stabilized by the relatively small lattice mismatch $(-2.0 \%)$ with the $\mathrm{Cu}$ and is unique for thin films since the inherent strain cannot be obtained in bulk Co. It is, however, interesting to check whether this strain is the only difference with the bulk fcc phase.

A sensitive tool for the study of thin Co film structural properties is ${ }^{59} \mathrm{Co}$ nuclear magnetic resonance (NMR). ${ }^{8,9}$ The NMR resonance frequencies, which are proportional to the magnetic hyperfine field at the Co nucleus, provide information on the distribution of Co atoms over different local environments. NMR can easily distinguish the various Co phases, observe impurities and interfaces, and can determine the strain in a Co film. An additional advantage is its ability to measure buried layers, which means that a film can be analyzed in its actual surroundings embedded in a structure.

So far NMR measurements have indeed identified all Co phases in thin-film structures. ${ }^{9}$ However, apart from the homogeneous strain in many layers (resulting in a small shift of the resonance frequency), some differences compared to bulk-Co spectra are worth noting. Most remarkable is the large linewidth usually observed in thin films. Whereas bulk fcc and hep materials have linewidths of about $0.7-1.0 \mathrm{MHz}$ (full width at half of the maximum intensity, FWHM), ${ }^{10,11}$ almost all thin Co films have linewidths of 5-10 MHz. A broadening of a NMR line can be caused by a direct influence of the interfaces on the hyperfine field ${ }^{12}$ or by the electric-field gradients (EFG's) caused by the deformation of the structure (see Sec. III C 3), but both effects are much too small to account for the $4-9 \mathrm{MHz}$ broadening. The large broadening in films is almost certainly caused by an inferior structural quality and a poor homogeneity as compared to the bulk material. This poor structural homogeneity precludes detailed studies on the properties of thin films.

In this paper we show that fct $\mathrm{Co}(001)$ grown by molecular-beam epitaxy (MBE) on $\mathrm{Cu}$ single crystals and covered with $\mathrm{Cu}$ presents a unique $\mathrm{Co}$ phase of bulklike structural quality. In order to optimize the quality, it is necessary to use single Co films instead of multilayers, which are certainly easier to measure, but are usually of lower structural quality simply due to accumulating defects. The (tetragonal) strain of the film is very homogeneous and shows only very little strain relief with increasing thickness contrary to the case of uncovered Co films. The elastic properties correspond to those of bulk fcc Co. Several effects of the strain are directly observable and measurable by NMR, which has not been possible in thin films before. A homogeneous EFG is observed and measured and can be quantitatively understood from the strain in the system. The (small) broadening of the NMR line compared to that of bulk fcc Co is shown to be due to effects of the noncubicity and not to an inferior quality. This means that the system actually represents a Co structure which is not existent in bulk (namely fct $\mathrm{Co}$ ), but has a structural quality and homogeneity comparable to a bulk crystal.

The paper is organized as follows: after providing the experimental details, we first compare Co layers on two types of $\mathrm{Cu}(001)$ substrates to determine which gives the optimum quality. Next we analyze the structure and strain of the best films by means of x-ray diffraction (XRD), scanning tunneling microscopy (STM), and low energy electron diffraction (LEED) and consecutively present our NMR results. These last are split in several parts: first the measurements of the hyperfine field are given, next the quadrupole splitting is 
analyzed, and finally the electric-field gradients are both determined from the measurement and compared with the values expected from the strain. In Sec. IV we compare our results with the literature and analyze the precise origin of the remaining NMR line broadening for these thin films relative to that of bulk fcc-Co. The paper ends with an overview of our conclusions.

\section{EXPERIMENTAL}

In order to obtain an optimum Co quality, the Co layers were single layers directly grown on a $\mathrm{Cu}(001)$ single crystal. This usually provides a better quality than a $\mathrm{Co} / \mathrm{Cu}$ multilayer structure. Contrary to the multilayer case the $\mathrm{Cu}$ will not be strained since its thickness is virtually infinite.

Preliminary to deposition, the $\mathrm{Cu}(001)$ single crystals were cleaned by sputter and anneal treatments until the surface was atomically flat and clean. The Co was MBE grown at a sample temperature of about $50{ }^{\circ} \mathrm{C}$, a background pressure below $10^{-10} \mathrm{mbar}$, and a growth rate of about $1 \mathrm{ML} / \mathrm{min}$. The Co thickness was controlled by an accurately calibrated quartz-crystal microbalance. Co thicknesses of $6,10,12,15,20,40,80$, and 280 monolayers (ML) were grown and the growth quality was checked in situ by LEED and STM. All Co layers were subsequently covered by $4 \mathrm{~nm}$ of $\mathrm{Cu}$ to prevent oxidation and to obtain optimally homogeneous Co structures with symmetric interfaces. The completed layer structures were analyzed ex situ by XRD and ${ }^{59}$ Co NMR. The NMR experiments were performed at $2 \mathrm{~K}$ in a home-built frequency-tuned spin-echo-NMR spectrometer. The sensitivity of the spectrometer is sufficient for measuring Co layers down to single ML thickness. ${ }^{13}$

As a preliminary investigation, we have also grown Co films on $100 \mathrm{~nm} \mathrm{Cu}$ buffer layers deposited on $\mathrm{Si}(001)$ substrates. The $\mathrm{Cu}$ buffer layer grows in a well-defined (001) orientation, although with an inferior quality than the $\mathrm{Cu}$ single crystals. Co films of 14, 28, 42, and $56 \mathrm{ML}$ were grown and covered with $4 \mathrm{~nm} \mathrm{Cu}$.

\section{RESULTS}

\section{A. $\mathrm{Cu}$ buffer layer versus $\mathrm{Cu}$ single crystal}

We first compare the structural quality of Co layers grown on the $\mathrm{Cu}(001)$ buffer layer with those grown on the $\mathrm{Cu}(001)$ single crystal. The quality on a strictly atomic scale can be evaluated from the NMR resonance linewidth. Such a measurement is not influenced by the better long-range order in the single crystal but is only sensitive to the local properties. Since the Co structures are nominally the same, any difference in the NMR linewidth can be directly related to the presence of local defects, stacking faults, or inhomogeneous strain in the films. A larger width directly indicates an inferior structural quality.

Co NMR spectra for films grown on a Cu buffer are plotted in Fig. 1(a) and the spectra for three films grown on a $\mathrm{Cu}$ single crystal in Fig. 1(b).

The spectrum of the thinnest Co film grown on a $\mathrm{Cu}$ buffer consists of a single line at $214.4 \mathrm{MHz}$, corresponding to Co in the fcc phase $(217 \mathrm{MHz}) .^{9}$ The shift of the reso-

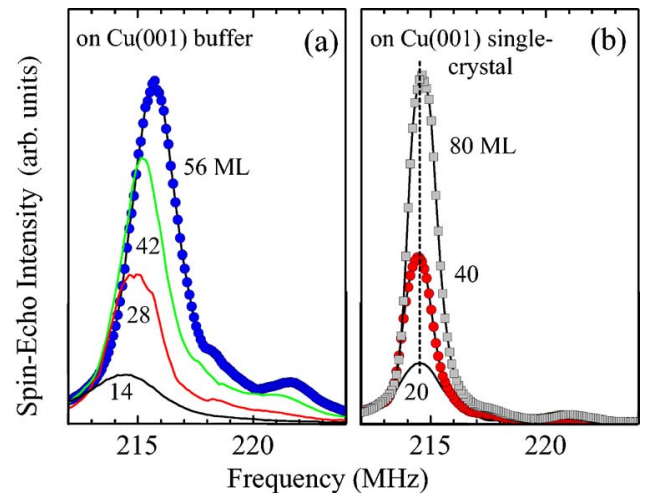

FIG. 1. (Color online) NMR spectra for $\mathrm{Co}(001)$ films grown on two different templates. (a) 14, 28, 42, and 56 ML thick Co films grown on $\mathrm{Si}(001) / 100 \mathrm{~nm} \mathrm{Cu}(001)$ and (b) 20, 40, and $80 \mathrm{ML}$ thick Co films grow on a $\mathrm{Cu}(001)$ single crystal. The spectra have been scaled in such a way that the area under the graphs is proportional to the Co thickness for (a) and (b) separately. The resonance frequency is proportional to the hyperfine field at the Co nuclei $(-10.054 \mathrm{MHz} / \mathrm{T})$.

nance line is caused by a small volume strain of the Co, which will be discussed more extensively in later sections.

With increasing film thickness the line gradually shifts to 215.7 MHz for the 56 ML Co film, indicating that a significant part of the strain is gradually relieved in this thickness range. Except for the thinnest film, all linewidths are about $2.5 \mathrm{MHz}$ FWHM, indicating a similar structural quality in all films and a homogeneous strain throughout the films.

The film strain stays homogeneous if strain relief occurs solely by means of misfit dislocations at the interface. This process is accompanied by stacking faults within the film, ${ }^{14,15}$ which are observed as the NMR lines at 219 and $222 \mathrm{MHz} .{ }^{9}$ The value for the strain relief derived from the measured stacking-fault density ${ }^{14}$ is comparable to the value determined from the NMR lineshift.

The spectra for 20, 40, and 80 ML Co films grown directly on a $\mathrm{Cu}(001)$ single crystal [Fig. 1(b)] do not show this strain relief as evinced by the constant position of the resonance line at $214.6 \mathrm{MHz}$ (very close to the frequency for the thinnest film on a $\mathrm{Cu}$ buffer) and the virtual absence of stacking faults. Additionally, the linewidth is strongly reduced (1.6, 1.2, and 1.1 MHz, respectively) compared to the films grown on a buffer. These linewidths are smaller than those of any other epitaxial thin Co film ${ }^{9}$ and only a few $100 \mathrm{kHz}$ larger than the widths observed for Co single crystals. ${ }^{10}$

Thus, Co layers on a $\mathrm{Cu}(001)$ single crystal show a very high-quality strained fcc phase with hardly any strain relief or stacking faults up to at least $80 \mathrm{ML}$. This in contrast to Co layers grown on a $\mathrm{Cu}(001)$ buffer layer, which are also of quite high quality, but show strain relief and associated stacking faults with increasing thickness. This means that Co layers grown on single-crystal substrates are much more suitable for the investigation of perfect thin films. We will exclusively investigate these in the remainder of this study. 


\section{B. Structure and strain}

First, the structure of the films and the strain in the films have been investigated as a function of thickness by means of STM, LEED, and XRD.

Our STM measurements show that for films up to $10 \mathrm{ML}$ thickness the Co grows in a nearly layer-by-layer mode as expected $^{3}$ and not more than three layers are observed simultaneously. For thicker films the roughness increases slightly: for a $20 \mathrm{ML}$ film and a $40 \mathrm{ML}$ film four and five layers are visible, respectively. Thus, although the growth is not in an ideal layer-by-layer fashion, it is quite close to it and the deviation is not expected to significantly influence the film structure.

LEED patterns show a square surface structure as expected for the fcc(001) direction without any thickness dependence even for still thicker films, indicating the absence of surface reconstructions and establishing the sole presence of the (001) orientation.

The $2 \%$ larger lattice parameter of $\mathrm{Cu}$ will lead to an expansion of the Co lattice in the plane of the film. This is accompanied by an out-of-plane contraction and results in a deformed fcc phase: face-centered-tetragonal (fct) Co. If, as usual, strain relief occurs at the interfaces, the strain can be described by a single parameter since the out-of-plane strain $\left(\epsilon_{\perp}=\Delta c / c_{0}\right)$, the volume strain $\left[\Delta V / V=\left(c_{0}+\Delta c\right)\left(a_{0}+\Delta a\right)^{2} /\right.$ $\left.\left(c_{0} a_{0}^{2}\right)-1\right]$, and the tetragonal deformation $(c / a)$ can all be calculated from the in-plane strain $\left(\epsilon_{\|}=\Delta a / a_{0}\right)$ by the standard elastic theory. ${ }^{16}$ Due to the very large thickness of the $\mathrm{Cu}$ single crystal only the Co film will be strained, with a maximum expansion for $\mathrm{Co}(001)$ of $\epsilon_{\|}=+1.99 \%$ at room temperature and $+2.00 \%$ at liquid He temperatures. ${ }^{17}$

The corresponding out-of-plane contraction in the fcc(001) orientations follows from ${ }^{16}$

$$
\epsilon_{\perp}=\gamma \epsilon_{\|}=-2 \frac{c_{12}}{c_{11}} \epsilon_{\|}
$$

Using $\quad c_{11}=(2.42 \pm 0.02) 10^{11} \mathrm{~N} / \mathrm{m}^{2} \quad$ and $\quad c_{12}$ $=(1.60 \pm 0.02) 10^{11} \mathrm{~N} / \mathrm{m}^{2}$, one finds $\gamma=-1.32 \pm 0.03$ for fcc Co at room temperature. ${ }^{18}$ Thus, a maximum out-of-plane strain $\epsilon_{\perp}=-(2.63 \pm 0.06) \%$ and a maximum volume strain $\Delta V_{\mathrm{Co}} / V_{\mathrm{Co}}=+(1.29 \pm 0.03) \%$ is expected for our Co films.

We have measured the out-of-plane lattice constant $c$ by means of LEED and XRD in order to determine the strain in our films.

For the top monolayers of uncovered Co films, $c$ can be determined from LEED I-V curves measured on the [00] spot. The energies at which the consecutive single-scattering peaks are found can yield a kinematical estimate of $c$. The accuracy of this analysis is, however, limited due to the presence of multiple scattering features. Also, the top-most uncovered Co ML's may have a lattice parameter somewhat deviating from the bulk value. ${ }^{19,20}$ The results are thus mainly useful for comparing the strain for different thicknesses.

For Co thicknesses of 10, 20, and $40 \mathrm{ML}, c$ $=(0.347 \pm 0.001) \mathrm{nm}$ is found, which corresponds to $\epsilon_{\perp}$ $=-(2.1 \pm 0.2) \%$. The $80 \mathrm{ML}$ Co film has $\epsilon_{\perp}=-1.6 \%$ and the $280 \mathrm{ML}$ film $\epsilon_{\perp}=-0.9 \%$. Apart from the absolute values, this indicates relaxation setting in somewhere between 40 and 80 ML and a still significant residual strain at $280 \mathrm{ML}$.

After deposition of a $4 \mathrm{~nm} \mathrm{Cu}$ film the completed structure was analyzed by XRD. Because of the small separation between the large peak from the $\mathrm{Cu}$ crystal and the $\mathrm{Co}(002)$ peak, only the samples with 80 and $280 \mathrm{ML}$ Co films could be measured reliably. A $\theta-2 \theta$ scan of the $80 \mathrm{ML}$ sample resulted in $c=(0.3458 \pm 0.0002) \mathrm{nm}$, i.e., in $\epsilon_{\perp}$ $=-(2.45 \pm 0.07) \%$ and $c / a=0.958$. This contraction corresponds to $(93 \pm 5) \%$ of the expected full strain and indicates only minimal strain relief. The out-of-plane correlation length calculated from the linewidth is close to $80 \mathrm{ML}$, indicating a homogeneous strain and a virtual absence of stacking faults within the film. This result differs from the LEED-IV result, which showed a significant relaxation in the $80 \mathrm{ML}$ film. However, this difference can easily be attributed to the $\mathrm{Cu}$ coverage, which stabilizes the strain of the Co film from the top side. Due to this, the strain in our films is more stable than in uncovered films, demonstrating the inapplicability of surface methods to determine the strain in buried layers.

On the other hand, a significant strain relief was found in the $\mathrm{Cu}$-covered $280 \mathrm{ML}$ film, where only $45 \%$ of the strain remained. As expected, ${ }^{14}$ this strain relief is accompanied by stacking faults within the film, leading to a reduced correlation length of about $110 \mathrm{ML}$. Strain relief was also observed after annealing of the $80 \mathrm{ML}$ film. This film showed $80 \%$ and $70 \%$ of the maximum strain after annealing at $300{ }^{\circ} \mathrm{C}$ and $400{ }^{\circ} \mathrm{C}$, respectively, both accompanied by some reduction in the correlation length.

Both the LEED and the XRD measurements have been performed at room temperature, as opposed to the NMR measurements that are performed at $2 \mathrm{~K}$. However, since the temperature dependence of the $\mathrm{Co}$ and $\mathrm{Cu}$ lattice parameters is very closely similar, no difference in the structure or the strain of the films is expected.

Thus, in our covered 80 ML Co film not more than $10 \%$ of the epitaxial strain is relaxed. This indicates that the strain relief is minor for all films up to this thickness, agreeing with the NMR results shown in Fig. 1 where no shift of the resonance line is observed for layers of 20, 40, and 80 ML thickness. Very thick films or annealed films do show a significant relaxation accompanied by a reduction of homogeneity. The films can be described as pure fct $\mathrm{Co}(001)$ since the (tetragonal) strain is homogeneous over the film and only a single orientation is present.

\section{NMR}

The fct phase is expected to have specific NMR properties that are exclusively observable if the structural quality is comparable to that of bulk fcc cobalt. Having established the presence of a stable and homogeneous fct phase (Sec. III B) with a very high structural quality (Sec. III A), we can test the observability of these properties. We will consecutively look at the direct influence of the strain on the Co hyperfine field, at the presence of quadrupole splitting due to the expected constant electric field gradients (EFG's) in the film, and at a measure of the film quality following from this. 


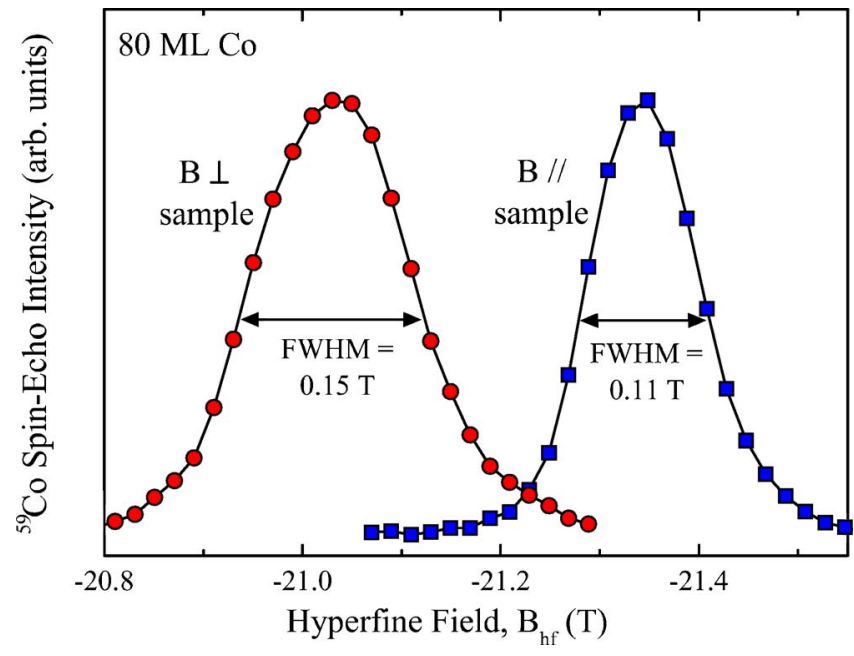

FIG. 2. (Color online) NMR spectra for a $\mathrm{Cu}(001) / 80 \mathrm{ML}$ $\mathrm{Co} / \mathrm{Cu}$ film, for both in-plane and out-of-plane saturation. Both the difference in the hyperfine fields and the difference in the linewidths are clearly observable.

Meanwhile we will analyze these features to check if they can provide extra information on the structure and strain in the film.

\section{Hyperfine fields}

An influence of the volume strain on the hyperfine field, $B_{\mathrm{hf}}$, is often observed in NMR. The enlarged volume of Co grown on $\mathrm{Cu}(001)$ leads to a reduced hyperfine field and thus a frequency shift. Unstrained fcc Co has a resonance frequency of $217.2 \pm 0.1 \mathrm{MHz}$ at liquid He temperatures $\left(B_{\mathrm{hf}}\right.$ $=-21.60 \mathrm{~T}) \cdot{ }^{10,13,21,22}$ The relative NMR frequency change is linearly proportional to the relative volume change: $\Delta f / f$ $=\Delta B_{\mathrm{hf}} / B_{\mathrm{hf}}=-(1.13 \pm 0.01) \Delta V / V$ at room temperature $23,46,47$ with $f$ the NMR frequency. At low temperatures the proportionality constant may be estimated to be $-1.16 \pm 0.06 .^{24}$

However, if the cubic symmetry is broken, the hyperfine field is anisotropic: $9,11 \quad B_{\mathrm{hf}}(\theta)=B_{\mathrm{hf}, \text { iso }}+\frac{1}{2} B_{\mathrm{hf}, \text { ani }}\left(3 \cos ^{2} \theta-1\right)$ with $B_{\mathrm{hf} \text {,iso }}$ and $B_{\mathrm{hf} \text {,ani }}$ the isotropic and anisotropic parts of the hyperfine field, respectively, and $\theta$ the angle with the symmetry axis, i.e., the film normal in our system. If the effects are independent, $B_{\mathrm{hf} \text {,iso }}$ is expected to change with the volume strain, as indicated above, while $B_{\mathrm{hf} \text {,ani }}$ may be expected to be roughly proportional to the tetragonal deformation $c / a$. Since, for maximum sensitivity, the thin-film NMR analysis is commonly performed without an applied field, ${ }^{9}$ the anisotropy of the hyperfine field is usually ignored as it can only be observed by means of applied fields.

In order to determine $B_{\mathrm{hf} \text {,ani, }}$, the samples were measured in a field of several Tesla applied both in the plane of the sample and perpendicular to the sample. For the perpendicular measurements the $1.80 \mathrm{~T}$ demagnetization field has to be taken into account. Resulting spectra for an $80 \mathrm{ML}$ Co film are plotted in Fig. 2. An anisotropy of $B_{\mathrm{hf} \text {,ani }}$ $=(+0.20 \pm 0.01) \mathrm{T}$ is found, which is not dependent on the thickness for films of 10, 20,40, and $80 \mathrm{ML}$. The anisotropy for the $280 \mathrm{ML}$ film and the $80 \mathrm{ML}$ film after annealing is significantly smaller, but could not be determined accurately enough for a quantitative comparison. The maximum anisotropy is about three times smaller than that of the hcp Co $\left(B_{\mathrm{hf}, \text { ani }}=+0.57 \mathrm{~T}\right)$.

$B_{\mathrm{hf} \text {,iso }}$ can be determined from the same measurements and a value of $(-21.24 \pm 0.01) \mathrm{T}$ (i.e., about $0.1 \mathrm{~T}$ smaller than the in-plane hyperfine field measured in zero applied field, where the difference is caused by the anisotropy) is found for all films up to $80 \mathrm{ML}$ thickness, corresponding to a constant volume strain of $\Delta V / V=(+1.44 \pm 0.08) \%$. We determine $\epsilon_{\|}$by combining the volume strain with $\epsilon_{\perp}=(-2.45 \pm 0.07) \%$ as determined by XRD (Sec. III B) and find $\epsilon_{\|}=(+1.95 \pm 0.08) \%$, confirming the almost full strain. The ratio $\gamma$ between $\epsilon_{\perp}$ and $\epsilon_{\|}$[Eq. (1)] is then $\gamma=(-1.26 \pm 0.04)$, in good agreement with the expected $\gamma=-1.32 \pm 0.03$.

Smaller strains are obtained for the $80 \mathrm{ML}$ film after annealing at $300{ }^{\circ} \mathrm{C}$ and $400{ }^{\circ} \mathrm{C}$ and for the $280 \mathrm{ML}$ film (in order of decreasing residual strain). Although for each of the films the value of $\gamma$ is in reasonable agreement with the expected value, there seems to be a small, linear dependence of the value of $\gamma$ on the residual strain in the film. Further measurements are necessary in order to analyze the significance and origin of this effect.

Thus, we have observed both a change and a significant anisotropy of the hyperfine field compared to the value for bulk fcc Co. The anisotropy is commonly ignored in the thin film NMR analysis, but should be taken into account when performing a quantitative strain analysis. The change of the hyperfine field in combination with the XRD measurements allows us to determine the elastic properties of the film.

\section{Quadrupole splitting}

A noncubic environment induces electric field gradients. A nucleus possessing a quadrupole moment (i.e., ${ }^{59} \mathrm{Co}$ ) interacts with these field gradients and a splitting of the NMR lines occurs. ${ }^{11}$ In most systems this splitting is too small to be observed directly by a spin-echo NMR experiment and will only lead to a broadening of the resonance line. This broadening will be anisotropic because the quadrupole splitting is anisotropic:

$$
\nu_{q}(\psi)=\nu_{q, \text { iso }}+\frac{1}{2} \nu_{q, \text { ani }}\left(3 \cos ^{2} \psi-1\right),
$$

with $\nu_{q \text {,iso }}$ and $\nu_{q \text {,ani }}$ the isotropic and anisotropic parts of the splitting and $\psi$ the angle with the symmetry axis, ${ }^{11}$ in our case the film normal. The isotropic part, the so-called localion contribution, is relatively small for Co (Refs. 11 and 25) and the anisotropic part which depends on the strain will be the major contribution.

In fec Co the total EFG is indeed known to be very small and equal to the isotropic part of the hcp-Co EFG, ${ }^{25}$ but for fct Co quite significant EFG's are expected dependent on the deviation from the cubic symmetry.

In Fig. 2 the resulting difference in the linewidth for the two magnetization directions can indeed be observed and is found to be $0.04 \mathrm{~T}$ (about $30 \%$ of the total width).

In principle, the quadrupole splitting can be directly estimated from this linewidth anisotropy, but an easier and more accurate method is available. A splitting of a resonance line 
into several evenly spaced components which are too close together to be resolvable can be observed as a modulation of the (otherwise exponential) spin-echo intensity decay. ${ }^{11,26-28}$ These modulations can only be observed if the EFG is homogeneous throughout the entire film since an inhomogeneous EFG will lead to a distribution of modulation frequencies. In thin films the EFG is usually not homogeneous enough and the effect has until now only been observed in bulk materials. For thin films only exponential or doubly exponential spin-echo decays have been reported up to now. ${ }^{9}$

In Fig. 3(a) the spin-echo intensity for a 20 ML fct-Co film is plotted as a function of the time $2 \tau$ between the first pulse and the echo. This figure shows a clear modulation of the echo decay (for comparison the corresponding unmodulated decay is also plotted) over more than one decade of intensity. This figure strongly resembles the spin-echo modulations observed for bulk hep Co by Fekete et al. ${ }^{11,29}$ although the damping in our case is significantly faster. This unique result is a direct proof that our films are of very high quality and much more homogeneous than any other thin Co film measured up to now by NMR.

The modulations can be fitted well by a slightly modified

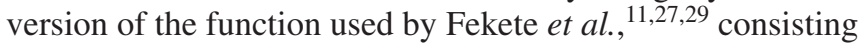
of exponentially decaying harmonics on an unmodulated background. In order to accurately fit our results a few additional terms to the formula are needed: a third harmonic term, slightly different decay times for each harmonic term and a doubly exponential overall decay as commonly observed in thin films. ${ }^{9}$ The resulting function is suitable to fit the curves for all different samples and parameters. Most fit parameters show only slight variations with the NMR frequency and pulse power. Due to the relatively fast damping, the uncertainty in the modulation frequency is a few percent (see Sec. III C 3).

The echo modulation is accompanied by the observation of multiple spin echoes, which have a different modulation behavior than the normal first echo as shown in Fig. 3(b). The second and third spin echoes have a two times and three times as high modulation frequency, respectively, and the second echo does not have an unmodulated off-set, exactly as predicted from the theory. ${ }^{27}$

The expected anisotropy of the modulation [following from Eq. (2)] is demonstrated in Fig. 3(c). Measurements on a $40 \mathrm{ML}$ instead of a $20 \mathrm{ML}$ Co film are shown because the signal to noise ratio decreases strongly on the application of an external field. $\nu_{q}$ for an in-plane applied field is closely similar to that of the zero-field measurements, indicating that the zero-field magnetization lies almost in-plane and that the NMR signal thus results from Co in domains and not in domain walls. ${ }^{9}$ The absolute frequency for an out-of-plane magnetization is about $50 \%$ higher than for an in-plane magnetization. This larger $\nu_{q}$ corresponds to the larger line width for that orientation (Fig. 2).

The observation of the modulations is a direct proof of the structural quality and the strength of the modulations can be used as a measure of the structural quality. By, for example, taking from the fit the number of modulations $\left(N_{\text {mod }}\right)$ with an amplitude larger than $10 \%$ of the unmodulated signal, this measure can be quantified. Since the modulation strength increases with the applied pulse power (linearly with

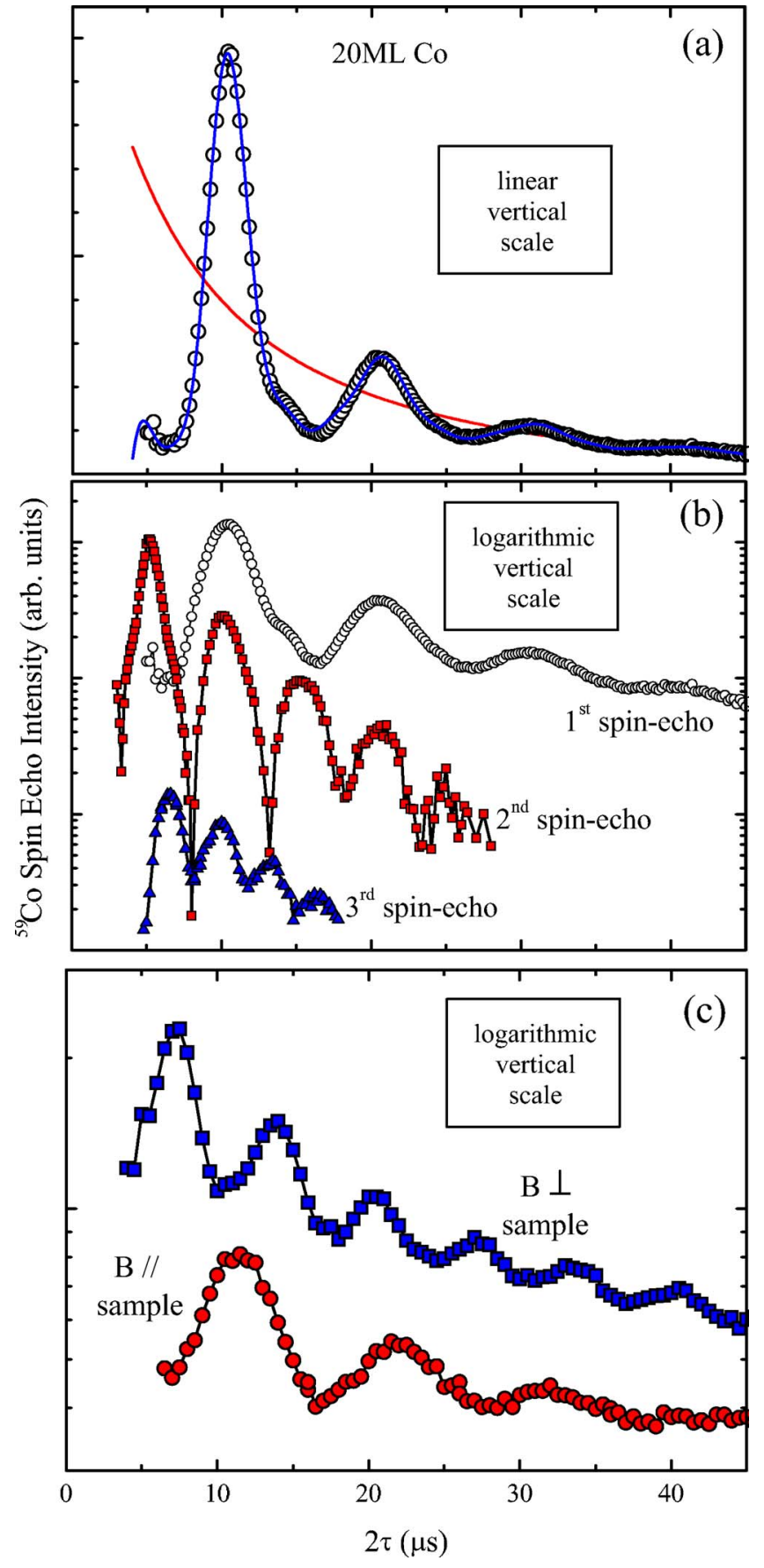

FIG. 3. (Color online) NMR spin-echo intensity vs the time between the first pulse and the echo. (a) The modulation observed for a $20 \mathrm{ML}$ Co film together with the fit and for comparison the corresponding unmodulated decay. (b) Multiple echoes are observed and show different modulation frequencies, as expected. (c) The modulation for a $40 \mathrm{ML}$ Co film, for a field applied either in-plane or out-of-plane, showing an anisotropy in the modulation frequency and thus in the quadrupole splitting. All spin-echo decays were measured approximately in the center of the NMR line, where the signal is the highest. (a) and (b) were measured in zero applied field and had their magnetizations roughly parallel to the sample plane. Note the different intensity scales. 
$0.19 \pm 0.02$ modulation per $\mathrm{dBm})$, this measure has to be taken at the optimum RF pulse power or corrected to that value. Our films have a constant $N_{\text {mod }}=3.0 \pm 0.1$. The 280 ML film, the annealed films as well as some $\mathrm{Co} / \mathrm{Cu}$ multilayers and films with thin Fe dusting layers on both sides have significantly lower values of $N_{\text {mod }}$, even though in some cases this difference cannot be seen in the linewidth or shows up only marginally in the XRD correlation length (Sec. III B), which demonstrates the sensitivity of the NMR to structural inhomogeneities. Nonetheless, the bulk hcp phase measured by Fekete et al. ${ }^{11}$ has an even better structural quality: $N_{\text {mod }}>7$.

Although this measure is somewhat dependent on the modulation frequency and may, for very thin films, be influenced by nonlocal effects, it has an additional advantage that it is also a measure of the disturbance of the regular NMR measurement by the modulations, indicating when care has to be taken with the interpretation of the exact line width and position. In our case, the presence of the modulations is the direct origin of the relatively low accuracy encountered in the previous sections.

Thus, we have indeed observed a well-defined quadrupole splitting in our films, both by means of an anisotropic broadening of NMR line and the observation of spin-echo decay modulations. The behavior of the modulations is similar to that in hep Co and multiple echoes and a clear anisotropy are also observed. The modulations cause complications in the regular NMR measurement, but their strength is a useful and sensitive measure of the structural quality of this type of film.

\section{Measurement of electric-field gradients}

Now that we have unambiguously demonstrated the presence of a well-defined and directly measurable electric-field gradient in our films, confirming the presence of a uniquely high-quality fct-Co phase, we can quantitatively analyze the information provided by these measurements. In the next two subsections we will investigate the quadrupole splitting and the corresponding EFG as a function of the strain in the film.

The quadrupole splitting in the in-plane direction (inplane or zero applied field) is constant from 15 to $80 \mathrm{ML} \mathrm{Co}$ thickness, as expected because of the closely similar strains, and is $\left|\nu_{q}\left(90^{\circ}\right)\right|=(191 \pm 3) \mathrm{kHz}$. Note that we cannot directly determine the sign of the splitting. An accurate value of a quadrupole splitting is best determined from a zero applied field measurement, which has a large signal to noise ratio.

However, because of its anisotropy, the quadrupole splitting has to be determined in two directions, necessitating the application of large fields. These measurements are much more noisy and less accurate, making it much harder to determine trustworthy values for each thickness. The damping is somewhat too fast to accurately link the modulation to the strain for the in-field measurements.

However, several general conclusions can be drawn. By applying Eq. (2), the quadrupole splitting can be separated into an isotropic and an anisotropic part. For both hep and fcc $\mathrm{Co}$, the isotropic part is known to equal $12.7 \%$ of the total hcp $\mathrm{EFG},{ }^{25}$ corresponding to a splitting of $\left|\nu_{q \text {,iso }}\right|$ $=(24.4 \pm 0.6) \mathrm{kHz}$. Although two of our films show strangely deviating values (up to $50 \mathrm{kHz}$ ), the other values indeed give $\left|\nu_{q \text {,iso }}\right|=(24 \pm 3) \mathrm{kHz}$. For the anisotropic part, we find values around $\left|\nu_{q \text {,ani }}\right|=(300 \pm 30) \mathrm{kHz}$, where the large variation is caused by the same two measurements that also show the deviating $\nu_{q \text {,iso }}$.

We find strong indications that our determination of $\nu_{q}\left(0^{\circ}\right)$ (out of plane) may be disturbed by the fast relaxation and the noise (since it has to be measured with an applied field). It shows nonsystematic variations for different samples while the in-plane modulation, which can be measured very accurately without a field, only shows gradual, systematic variations with thickness or annealing.

Assuming that indeed the out-of-plane component is disturbed for two of the measurements, we find $\left|\nu_{q \text {,iso }}\right|$ $=(24 \pm 3) \mathrm{kHz}$ for all samples and $\left|\nu_{q \text {,ani }}\right|=340,334$, and $328 \mathrm{kHz}$ for the 20,40 , and $80 \mathrm{ML}$ films, respectively, corresponding to a very small and gradual strain relief. Annealing of the $80 \mathrm{ML}$ film at $300{ }^{\circ} \mathrm{C}$ and $400{ }^{\circ} \mathrm{C}$ leads to 270 and $245 \mathrm{kHz}$ respectively, corresponding to the significant relaxation of these films seen in Sec. III C 1.

The anisotropic part of the electric-field gradient follows from the quadrupole splitting by $\nu=\left(3 e Q V_{z z}\right) /[2 I(2 I-1) h]$, with $Q$ the quadrupole moment. The $328 \mathrm{kHz}$ anisotropic splitting of the $80 \mathrm{ML}$ film, thus corresponds to an EFG of $\left|V_{z z}^{\text {ani }}\right|=(4.61 \pm 0.06) 10^{18} \mathrm{~V} / \mathrm{m}^{-2}$.

Although the accuracy of the out-of-plane quadrupole splitting is sometimes doubtful due to the fast relaxation and noise, the expected value of the isotropic splitting is reproduced. By using this value, the anisotropic splittings can be accurately determined from the zero-field measurements alone.

\section{Calculation of electric-field gradients}

We will now compare our results with the EFG's originating from the strain in the film.

The EFG at a nucleus arises from the distribution of the charge around it. The anisotropic part used to be calculated by viewing the crystal as composed of positive point charges at the lattices sites, together with a uniform compensating electron charge and calculating the so-called lattice contribution by direct summation over the lattice sites. ${ }^{11}$ The real anisotropic splitting was found to be directly proportional to this lattice contribution for a remarkably wide range of systems. ${ }^{30}$ Although this result is not ideal, since large empirical antishielding factors have to be used and since actually full electronic calculations should be used, ${ }^{31}$ lattice summation is still a useful way for determining the influence of strain within one specific system.

The direct summation over the lattice sites of a homogeneous fct film reproduces the results by De Wette. ${ }^{33}$ The influence of the finite film thickness can be neglected since electric fields and their gradients are shielded within a few atomic distances. ${ }^{34}$ The result is given in Fig. 4.

In our case this lattice summation is very suitable since we can directly compare our values with the known quadrupole splitting for hcp Co. ${ }^{11}$ Our only assumption is the equality of the universal correlation constant for fcc and hep Co, ${ }^{30}$ which is very reasonable since this correlation is indeed universal over most systems. With this assumption the ratio be- 


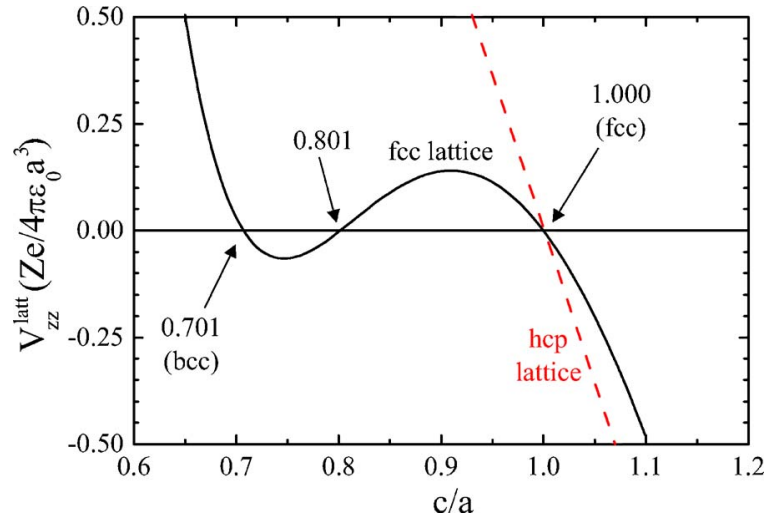

FIG. 4. (Color online) Calculated lattice term to the electricfield gradient (EFG), to which the real EFG is expected to be proportional, both for a fcc/fct lattice and an hep lattice as a function of the strain. The resulting EFG has units $V \mathrm{~m}^{-2}$ and $Z$ is the ionic charge (Ref. 32). Note that for hcp a reduced $c / a$ is taken, where $c / a=1$ corresponds to unstrained hcp.

tween the anisotropic quadrupole splitting for fct and hcp Co is equal to the ratio between the lattice contributions for both systems and, because the lattice contribution for our fct system is the only unknown of these four, we can determine it without having to go into the details of the physically somewhat dubious antishielding factors.

The hep Co anisotropic quadrupole splitting is $170 \mathrm{kHz}$ and the lattice contribution $V_{z z}^{\text {latt }}(\mathrm{hcp})=(\mathrm{Ze}) /\left(4 \pi \epsilon_{0} a^{3}\right)$ $\times 0.0548 .{ }^{11,25}$ For our $80 \mathrm{ML}$ film the quadrupole splitting is 1.9 times as large and thus $V_{z z}^{\text {latt }}(\mathrm{fct})=(\mathrm{Ze}) /\left(4 \pi \epsilon_{0} a^{3}\right)$ $\times(0.106 \pm 0.002)$. From the calculations represented in Fig. 4 follows $(c / a-1)=-(4.65 \pm 0.3) \%$ corresponding to $\epsilon_{\|}=\Delta a / a=+(2.0 \pm 0.1) \%$ [Eq. (1)]. This result is in excellent agreement with the measured strain (Sec. III C 1) of $\epsilon_{\|}=\Delta a / a=+(1.95 \pm 0.1) \%$.

The slight decrease in the quadrupole splitting for increasing the Co thickness from 20 to $80 \mathrm{ML}$ would correspond to a strain relief of about $3 \%$ which is not significant in view of the experimental errors. There may indeed be some strain relief in this range, ${ }^{5}$ but if there is, it is only very minor. The decrease in the EFG for the annealed $80 \mathrm{ML}$ films (to 75\% and $65 \%$ respectively) agrees roughly with the decrease in strain as seen from the NMR line shift and the XRD results.

Thus, our measurement of the EFG agrees well with the measured strains. For Co thicknesses of $12 \mathrm{ML}$ and less, the modulation frequency increases slightly and for very thin films (6 or $7 \mathrm{ML} \mathrm{Co}$ ) decreases steeply. We attribute these effects to the influence of the line splitting due to the interfaces, ${ }^{12}$ which become observable at the same thicknesses, and to possible influences from the interfaces directly on the EFG. ${ }^{34}$ This is corroborated by the observation of small changes in the EFG as a function of the NMR frequency in a 15 ML Co film. Since the different frequencies correspond to different distances from the interface, ${ }^{12}$ this means that nonlocal effects on the EFG are indeed observed.

We have shown by a very simple method that the strain corresponding to our measured EFG agrees excellently with the strain determined in Sec. III B. For extremely thin films we can observe direct influences from the interfaces, however, we cannot measure them accurately enough for a quantitative analysis.

\section{DISCUSSION}

We will now compare the structural results, the EFG measurement method, and the measured EFG with reports in the literature. After that, we will analyze why the linewidth of our films is larger than that of the bulk fcc Co even though the structural quality is expected to be comparable.

Our results on the development of strain in the films obtained by LEED and XRD (Sec. III B) differ in some respects from those published in the literature. Many studies have been performed on the growth of the first few ML of Co on $\mathrm{Cu}(001)$ or on the growth of $\mathrm{Co} / \mathrm{Cu}$ multilayers. The number of studies on the growth of single Co films thicker than about $10 \mathrm{ML}$ is quite limited. Most studies find almost layer-by-layer growth above 2 ML thickness and up to at least $20 \mathrm{ML}^{35}$ However, the values for the strain vary somewhat ${ }^{19,36,37}$ and the distinction between polycrystalline and single-crystalline elastic constants is not always made. Strains are determined by LEED and the variation in results seems to confirm that care has to be taken with this kind of measurement when using it to obtain absolute values of the lattice parameter for films thicker than a few ML.

Studies of even thicker layers have been performed by Weber et al. ${ }^{5}$ and Schindler et al. ${ }^{38}$ Schindler et al. found the presence of two Co phases with different strains for films thicker than $10 \mathrm{ML}$, which may be specific for the electrodeposition method used. Weber et al. measured the inplane lattice spacing of MBE-grown Co films up $40 \mathrm{ML}$ thickness on $\mathrm{Cu}(001)$ by reflection high-energy electron diffraction (RHEED). They found a fully strained phase up to 10-15 ML of Co depending on the growth temperature and above that thickness an exponential relaxation to a (extrapolated) residual strain of about $70-80 \%$ of the maximum strain. This result corresponds to ours in the prediction of large residual strains for thick films $(80-85 \%$ of the full strain for 40-80 ML Co films). In our case, the residual strain in this range is even higher due to the influence of the $\mathrm{Cu}$ top layer. The observed strain relief reported for uncovered Co layers is partly a reversible property of Co surface layers, which may be undone by subsequent $\mathrm{Cu}$ coverage. However, the strong strain relief in our thickest film is an irreversible event, different from the extrapolated behavior of the thinner films. ${ }^{5}$

Several other methods are used in the literature to determine the EFG's. The easiest is continuous wave NMR which has a high enough resolution to directly resolve the splitting in one of the orientations in hep Co. ${ }^{28}$ Its sensitivity is, however, too low to be applied to thin films.

Most EFG measurements employ radiative detection from oriented radioactive nuclei implanted in a metal matrix, e.g., perturbed angular correlation spectroscopy (PACS), nuclear magnetic resonance on oriented nuclei (NMR-ON) and modulated adiabatic passage on oriented nuclei (MAPON).${ }^{39-42}$ Especially MAPON is very sensitive even to small EFG's and has been successfully used to determine the 
EFG of Co in unstrained fcc Co. ${ }^{25}$ It has moreover the advantage that the EFG distribution and sign can be determined directly. However, the implantation of radioactive ions in our thin films may significantly influence their properties and quality. An alternative might be the irradiation of the layer with thermal neutron in order to produce the necessary ${ }^{60} \mathrm{Co}$, which may be expected to cause much less damage. This analysis might be a useful object for future MAPON studies.

Hardly any studies have been performed concerning the influence of the strain within a single material (as opposed to the many studies concerning the hcp metals with different $c / a$ ratios) and those performed do not present a quantitative analysis. ${ }^{43,44}$ Thus, we cannot compare our results on the influence of strain directly with other experimental papers.

We can, however, compare the isotropic part of the EFG in our system with that in other Co systems. Although for specific impurity atoms, an EFG depending on the orientation with respect to the different crystallographic axes has been found, ${ }^{45}$ only an axially symmetric lattice contribution and an isotropic local ion contribution have been observed for Co. ${ }^{11,25}$ For fcc Co the lattice contribution is absent (due to the cubic symmetry) and the observed EFG is equal to the isotropic part in hcp Co. This agrees with the expected additivity of the lattice and local ion EFG's. We find the same absolute value for this isotropic EFG, however, of opposite sign. For hcp Co the isotropic and anisotropic parts have equal sign, for fct Co we find them to have opposite sign. This sign change can be derived directly by means of the ratio between $\nu_{q}\left(0^{\circ}\right)$ (out of plane) and $\nu_{q}\left(90^{\circ}\right)$ (in plane) and Eq. (2): if the ratio between $\nu_{q}\left(0^{\circ}\right)$ and $\nu_{q}\left(90^{\circ}\right)$ is larger than 2 then $\nu_{q \text {,iso }}$ has the same sign as $\nu_{q \text {,ani }}$, while if the ratio is smaller than 2 the signs are opposite. Since the anisotropic lattice contribution, $v_{q \text {,ani }}$, has the same sign for hcp Co and fct Co (both have a $c / a$ ratio below the symmetric value, see Fig. 4), this means that the local ion contribution has changed signs in this system. Although we can only accurately determine the quadrupole splitting without an applied field, our field measurements are still accurate enough to reliably determine these signs. It is not clear what causes this effect, but maybe the additivity of lattice and local ion contribution is somewhat less straightforward after all. Further measurements on other systems, together with a comparison with electronic-structure calculations for these systems, are necessary to study the origin of this effect.

Finally, we will analyze the linewidth in our films. The observation of well-defined EFG's tells us that our films are very homogeneous. Such a film should have an NMR linewidth comparable to that of high-quality bulk material. However, several effects inherent to strained thin films influence our linewidth and have to be taken into account when comparing widths.

As a typical example, we take our 20 ML Co film, which has a linewidth in zero field of $0.19 \mathrm{~T}$ FWHM, where for accuracy reasons the FWHM is determined from the width of a Gaussian fit of the entire line. This value is significantly larger than the $0.07 \mathrm{~T}$ found for bulk fcc films.

However, several effects inherent to the thin fct films are responsible for most of this broadening. The anisotropic hyperfine field causes $0.03 \mathrm{~T}$ broadening in a zero-field measurement compared to a measurement with an applied field in-plane, due to the slight distribution around the in-plane magnetization without an applied field. Secondly, the interfaces cause a broadening by a direct and long-ranging modulation of the hyperfine field per monolayer. ${ }^{12}$ In very thin films the second, third, and fourth ML from an interface are resolvable as satellites while for the further ML's there is a very slowly decaying modulation on the hyperfine field. Ignoring the satellites, which are hard to see in a thicker film, during the fitting, results in an overestimation of the linewidth by $0.02 \mathrm{~T}$. Also the small modulation causes a broadening of the NMR line by about $0.02 \mathrm{~T}$, as seen from the simulation. In the third place there is the quadrupole splitting, which causes a broadening of $0.03 \mathrm{~T}$ in a parallel orientation and of $0.07 \mathrm{~T}$ in a perpendicular orientation (using seven Gaussian lines with intensity ratios 7, 12, 14, 15, 14, 12 , and 7, following from the Clebsch-Gordan coefficients). The basic linewidth, without any of these effects is $0.09 \mathrm{~T}$, not much higher than that of bulk fcc Co and the anisotropy of $0.04 \mathrm{~T}$ agrees well with the measured value. Similar results are found for both thinner and thicker films.

We can thus conclude that hardly any broadening due to defects, local strain variations, or long range effects of dislocations is present and that we can understand both the line width and the line width anisotropy quantitatively.

\section{CONCLUSIONS}

We have grown thin Co films on $\mathrm{Cu}(001)$ single-crystal substrates by MBE. These films are found to be in a homogeneously-strained fct phase. Films up to $80 \mathrm{ML}$ show more than $90 \%$ residual strain relative to perfectly lattice matched $\mathrm{Co}$ on $\mathrm{Cu}(001)$. By combining XRD and NMR measurements, we find that the behavior of the Co is well described by the elastic theory. The somewhat larger strain in our systems compared to earlier results in the literature ${ }^{5}$ is probably caused by the $\mathrm{Cu}$ coverage layer, emphasizing the significant difference between uncovered and covered thin films.

We observe a breakdown of the gradual strain relief for films of several hundred $\AA$, which have a much smaller residual strain than predicted by extrapolation. The large residual strain only occurs for direct growth on $\mathrm{Cu}$ single crystals, growth on $\mathrm{Cu}$ buffer layers results in a strong gradual strain relief that decreases the structural quality.

The NMR linewidth of these films is comparable to that found in bulk materials, i.e., an order of magnitude smaller than usually found for thin fcc or fct films, confirming the virtual absence of inhomogeneities, defects, and dislocations.

We have directly observed the influence of the tetragonal Co deformation on the hyperfine field by NMR. This has not been possible before since a well-defined fct phase can only be stabilized in thin films and these never had the requisite quality up to now. The deformation results in an anisotropic hyperfine field, an anisotropic linewidth, and a well-defined anisotropic quadrupole splitting which is observed by modulations of the spin-echo decay. The electric-field gradient in the film, which is responsible for the quadrupole splitting, agrees well with the value expected from the strain. The observability of this effect indicates an extremely high qual- 
ity and homogeneity in the thin film structure. The strength of the modulation can be used as a quantitative measure of the film quality. For very thin films (thinner than 15 ML) a slight position dependence of the EFG is observed due to a small direct influence from the interfaces.

We can conclude that fct $\mathrm{Co}$ on $\mathrm{Cu}(001)$ is a very welldefined phase with an almost thickness independent strain and structural quality up to at least $80 \mathrm{ML}$ Co. This system, in principle, provides an extra Co phase (next to hcp, fcc, and bcc) that may, for example, be used in spintronic model systems for studying the influence of band structure and electronic properties by varying the phase of the magnetic electrode. Furthermore, nuclear magnetic resonance and especially the measurement of electric-field gradients have been shown to be an ideal tool for determining the local structure and its homogeneity and quality in thin Co films.
*Electronic address: j.t.kohlhepp@tue.nl

${ }^{1}$ E. Y. Tsymbal, O. N. Mryasov, and P. R. Leclair, J. Phys.: Condens. Matter 15, R109 (2003).

${ }^{2}$ S. A. Wolf, D. D. Awschalom, R. A. Buhrman, J. M. Daughton, S. von Molnár, M. L. Roukes, A. Y. Chtchelkanova, and D. M. Treger, Science 294, 1488 (2001).

${ }^{3}$ A. K. Schmid and J. Kirschner, Ultramicroscopy 42, 483 (1992).

${ }^{4}$ E. Navas, P. Schuster, C. M. Schneider, J. Kirschner, A. Cebollada, C. Ocal, R. Miranda, J. Cerdá, and P. de Andrés, J. Magn. Magn. Mater. 121, 65 (1993).

${ }^{5}$ W. Weber, A. Bischof, R. Allenspach, C. H. Back, J. Fassbender, U. May, B. Schirmer, R. M. Jungblut, G. Güntherodt, and B. Hillebrands, Phys. Rev. B 54, 4075 (1996).

${ }^{6}$ E. A. M. van Alphen, A. H. J. Colaris, S. K. J. Lenczowski, H. A. M. de Gronckel, C. Schönenberger, and W. J. M. de Jonge, J. Magn. Magn. Mater. 156, 29 (1996).

${ }^{7}$ G. J. Strijkers, J. T. Kohlhepp, H. J. M. Swagten, and W. J. M. de Jonge, Appl. Magn. Reson. 19, 461 (2000).

${ }^{8}$ W. J. M. de Jonge, H. A. M. de Gronckel, and K. Kopinga, in Ultrathin Magnetic Structures II, edited by B. Heinrich and J. A. C. Bland (Springer-Verlag, Berlin, 1994), pp. 279-290.

${ }^{9}$ P. C. Riedi, T. Thomson, and G. J. Tomka, in Handbook of Magnetic Materials, edited by K. H. J. Buschow (Elsevier, Amsterdam, 1999), Vol. 12, pp. 97-258.

${ }^{10}$ T. Thomson, P. Riedi, C. Morawe, and H. Zabel, J. Magn. Magn. Mater. 156, 89 (1996).

${ }^{11}$ D. Fekete, H. Boasson, A. Grayevski, V. Zevin, and N. Kaplan, Phys. Rev. B 17, 347 (1978).

${ }^{12}$ H. Wieldraaijer, W. J. M. de Jonge, and J. T. Kohlhepp, Phys. Rev. Lett. 93, 177205 (2004).

${ }^{13}$ G. J. Strijkers, Ph.D. thesis, Eindhoven University of Technology (1999).

${ }^{14}$ J. W. Matthews, Thin Solid Films 5, 369 (1970).

${ }^{15}$ P. Schall, I. Cohen, D. A. Weitz, and F. Spaepen, Science 305, 1944 (2004).

${ }^{16}$ R. P. Feynman, R. B. Leighton, and M. Sands, The Feynman Lectures on Physics (Addison-Wesley, Reading, 1963), Vol. 2.

${ }^{17} \mathrm{We}$ use for the lattice constants of fcc $\mathrm{Co}$ and $\mathrm{Cu}: a_{\mathrm{Co}}$ $=0.35445 \mathrm{~nm}, a_{\mathrm{Cu}}=0.3615 \mathrm{~nm}$ at room temperature, and $a_{\mathrm{Co}}$ $=0.3532 \mathrm{~nm}$ and $a_{\mathrm{Cu}}=0.3604 \mathrm{~nm}$ at liquid He temperature (Ref. 18).

${ }^{18}$ Numerical Data and Functional Relationships in Science and Technology, Landolt-Börnstein, New Series, Group III, Vols. 18 and 19 (Springer-Verlag, Berlin, 1984).

${ }^{19}$ J. R. Cerdá, P. L. de Andres, A. Cebollada, R. Miranda, E. Navas, P. Schuster, C. M. Schneider, and J. Kirschner, J. Phys.: Condens. Matter 5, 2055 (1993).
${ }^{20}$ S. Müller, A. Kinne, M. Kottcke, R. Metzler, P. Bayer, L. Hammer, and K. Heinz, Phys. Rev. Lett. 75, 2859 (1995).

${ }^{21}$ M. Kawakami, T. Hihara, Y. Kōi, and T. Wakiyama, J. Phys. Soc. Jpn. 33, 1591 (1972).

${ }^{22}$ H. Brömer and H. L. Huber, J. Magn. Magn. Mater. 8, 61 (1978).

${ }^{23}$ The frequency shift has been found to be linearly related to an applied isotropic pressure (Refs. 46 and 47) with $\partial \ln f / \partial p$ $=(6.01 \pm 0.01) \times 10^{-12} \mathrm{~Pa}^{-1}$, with $p$ the applied pressure. Since the volume change is also linearly proportional to the applied pressure $\quad\left(\partial \ln V / \partial p=\mathrm{K}^{-1}=3\left(c_{11}+2 c_{12}\right)^{-1}=(5.35 \pm 0.06)\right.$ $\times 10^{-12} \mathrm{~Pa}^{-1}$, with $K$ the bulk modulus), a linear relation exists between the change in NMR frequency and the change in volume: $\Delta f / f=\Delta B_{\mathrm{hf}} / B_{\mathrm{hf}}=-(1.13 \pm 0.01) \Delta V / V$.

${ }^{24}$ At low temperatures the elastic constants $c_{11}$ and $c_{12}$ are a few percent larger (Ref. 18).

${ }^{25}$ W. D. Hutchison, A. V. J. Edge, N. Yazidjoglou, and D. H. Chaplin, Phys. Rev. Lett. 67, 3436 (1991).

${ }^{26}$ E. L. Hahn and D. E. Maxwell, Phys. Rev. 88, 1070 (1952).

${ }^{27}$ H. Abe, H. Yasuoka, and A. Hirai, J. Phys. Soc. Jpn. 21, 77 (1966).

${ }^{28}$ H. Enokiya, J. Phys. Soc. Jpn. 42, 796 (1977).

${ }^{29}$ Please note that there are two typographical errors in Fekete et al. (Ref. 11): the independent parameter in Fig. 2 is wrongly given as $2 \tau$, while it actually is $\tau$ and a factor of $\pi$ is missing within both harmonic terms in Eq. (8).

${ }^{30}$ P. Raghavan, E. N. Kaufmann, R. S. Raghavan, E. J. Ansaldo, and R. A. Naumann, Phys. Rev. B 13, 2835 (1976).

${ }^{31}$ P. Blaha, K. Schwarz, and P. H. Dederichs, Phys. Rev. B 37, 2792 (1988).

${ }^{32}$ Most publications use CGS units and thus have the EFG, $q_{\text {latt }}$, in $\mathrm{cm}^{-3}$ and the first fraction as $Z / a^{3}$.

${ }^{33}$ F. W. de Wette, Phys. Rev. 123, 103 (1961).

${ }^{34}$ J. Korecki and U. Gradmann, Phys. Rev. Lett. 55, 2491 (1985).

${ }^{35}$ H. Li and B. P. Tonner, Surf. Sci. 237, 141 (1990).

${ }^{36}$ A. Clarke, G. Jennings, R. F. Willis, P. J. Rous, and J. B. Pendry, Surf. Sci. 187, 327 (1987).

${ }^{37}$ O. Heckmann, H. Magnan, P. le Fevre, D. Chandesris, and J. J. Rehr, Surf. Sci. 312, 62 (1994).

${ }^{38}$ W. Schindler, T. Koop, A. Kazimirov, G. Scherb, J. Zegenhagen, T. Schultz, R. Feidenhans'1, and J. Kirschner, Surf. Sci. 465, L783 (2000).

${ }^{39}$ R. S. Raghavan, P. Raghavan, and E. N. Kaufmann, Phys. Rev. Lett. 31, 111 (1973).

${ }^{40}$ E. Matthias and R. J. Holliday, Phys. Rev. Lett. 17, 897 (1966).

${ }^{41}$ E. Hagn, Phys. Rev. B 25, 1521 (1982).

${ }^{42}$ P. T. Callaghan, P. J. Back, and D. H. Chaplin, Phys. Rev. B 37, 4900 (1988). 
${ }^{43}$ G. R. Hoy and K. P. Singh, Phys. Rev. 172, 514 (1968).

${ }^{44}$ R. C. Mercader and T. E. Cranshaw, J. Phys. F: Met. Phys. 5, L124 (1975).

${ }^{45}$ G. Seewald, E. Hagn, E. Zech, D. Forkel-Wirth, A. Burchard, and ISOLDE Collaboration, Phys. Rev. Lett. 78, 1795 (1997).
${ }^{46}$ R. V. Jones and I. P. Kaminow, Bull. Am. Phys. Soc. 5, 175 (1960), abstract only.

${ }^{47}$ D. H. Anderson and G. A. Samara, J. Appl. Phys. 35, 3043 (1964). 